\section{Does the use of bedside ultrasonography reduce emergency department length of stay for patients with renal colic?: a pilot study}

\author{
Yong Hoon Park', Ru Bi Jung' ${ }^{1}$ Young Geun Lee', Chong Kun Hong², \\ Jung-Hwan Ahn', Tae Yong Shin', Young Sik Kim'1, Young Rock Ha' \\ 'Department of Emergency Medicine, Daejin Medical Center, Bundang Jesaeng General Hospital, \\ Seongnam, Korea \\ ${ }^{2}$ Department of Emergency Medicine, Hyundae General Hospital, Namyangju, Korea \\ ${ }^{3}$ Department of Emergency Medicine, Ajou University School of Medicine, Suwon, Korea
}

Objective The aim of this study was to evaluate the effect of adding bedside ultrasonography to the diagnostic algorithm for nephrolithiasis on emergency department (ED) length of stay.

Methods A prospective, randomized, controlled pilot study was conducted from October 2014 to December 2014 with patients with acute flank pain. In the non-ultrasonography group (NUSG), non-contrast computed tomography was selected based on clinical features and hematuria in the urinalysis. In the ultrasonography group (USG), non-contrast computed tomography was selected based on clinical features and hydronephrosis on bedside ultrasonography. The primary outcome was ED length of stay. The secondary outcomes were radiation exposure, amount of analgesics, proportion of patients with diseases other than ureteral calculus, and proportion of patients with unexpected ED revisits within 7 days from the index visit.

Results A total of 103 patients were enrolled (NUSG, 51; USG, 52). The ED length of stay for the USG (89.0 minutes) was significantly shorter than that for the NUSG (163.0 minutes, $\mathrm{P}<0.001)$. There were no significant differences between the two groups in the radiation exposure dose (5.29 and $5.08 \mathrm{mSv}$, respectively; $P=0.392)$, amount of analgesics $(\mathrm{P}=0.341)$, proportion of patients with diseases other than ureteral calculus ( $13.0 \%$ and $6.8 \%$, respectively; $P=0.486)$, and proportion of patients with unexpected ED revisits within 7 days from the index visit $(7.8 \%$ and $9.6 \%$, respectively; $P=1.000$ ).

Conclusion The use of early bedside ultrasonography for patients with acute flank pain could reduce the $E D$ length of stay without increasing unexpected $E D$ revisits.

Keywords Length of stay; Ultrasonography; Ureterolithiasis

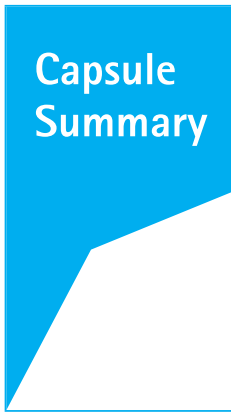

elSSN: 2383-4625

Received: 2 June 2016

Revised: 29 August 2016

Accepted: 12 September 2016

Correspondence to: Young Rock Ha Department of Emergency Medicine, Daejin Medical Center, Bundang Jesaeng General Hospital, 20 Seohyeon-ro 180beon-gil, Bundanggu, Seongnam 13590, Korea E-mail:youngrock.ha@gmail.com

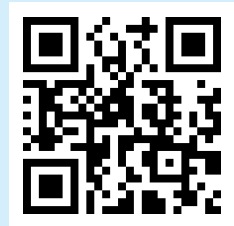

How to cite this article: Park YH, Jung RB, Lee YG, Hong CK, Ahn JH, Shin TY, Kim YS, Ha YR. Does the use of bedside ultrasonography reduce emergency department length of stay for patients with renal colic?: a pilot study. Clin Exp Emerg Med 2016;3(4):197-203.

This is an Open Access article distributed under the terms of the Creative Commons Attribution Non-Commercial License (http:// creativecommons.org/licenses/by-nc/4.0/). 


\section{INTRODUCTION}

Renal colic is a common cause of emergency department (ED) visits. ${ }^{1}$ Renal colic is a clinical syndrome that presents with acute cyclical flank pain and is most commonly due to nephrolithiasis. ${ }^{2}$ The prevalence of nephrolithiasis has increased over time; it is experienced by $3 \%$ to $15 \%$ of adults within their lifetime and is detected in about $2 \%$ of adult ED patients. ${ }^{3,4}$

Nephrolithiasis is initially diagnosed based on clinical presentation followed by imaging studies. ${ }^{2}$ The use of bedside ultrasonography (BUS) for renal colic has increased in recent years. ${ }^{2}$ Its advantage over other diagnostic imaging modalities is that it has no radiation hazards. ${ }^{2}$ However, it is less sensitive than computed tomography (CT) for identifying the size and number of renal stones and rarely identifies the location of a renal stone; many patients who initially undergo BUS will need a CT scan to confirm the presence and location of the stone. ${ }^{5,6}$ Non-contrast CT has higher sensitivity and specificity than BUS; hence, it has been considered the gold standard for the diagnosis of nephrolithiasis.-9

However, the decision to perform CT based only on clinical features results in nonspecific CT results for some patients with ongoing flank pain. Hematuria is the single most discriminating predictor of a renal stone in patients with flank pain. ${ }^{10}$ Hence, many physicians perform CT based on the combination of clinical features and hematuria on urinalysis. ${ }^{11}$ Unfortunately, patients with flank pain commonly present with dysuria and are sometimes unable to urinate; therefore, urinalysis results frequently require more time than expected. ${ }^{8}$ Because rapid diagnosis in the ED can facilitate the most appropriate therapy, the ED stay is prolonged while waiting for the urinalysis results. All of these factors can affect the ED strategy, diagnostic time, and proper treatment. BUS has high specificity but it is not as sensitive for detecting hydronephrosis. ${ }^{12}$

The aim of this study was to evaluate the effect on ED length of stay of adding early BUS to the diagnostic algorithm for nephrolithiasis.

\section{METHODS}

\section{Study design and ethics}

This prospective, randomized, controlled pilot study was conducted with consecutive patients with flank pain. This study was approved by the institutional review board (EMC 13-03), and informed consent was obtained from all patients. When a patient was unable to sign the consent form due to distress, we obtained consent from guardians.

\section{Setting}

This study was conducted in the ED of an urban secondary care hospital in Gyeonggi-do province, South Korea; the ED cares for about 35,000 patients annually.

\section{Study protocol}

Non-ultrasonography group

If the treating physician thought that the patient's clinical presentation was compatible with nephrolithiasis, we immediately administered parenteral analgesics and awaited the result of urinalysis. If urinalysis revealed microscopic hematuria, non-contrast helical CT (NECT) was performed. When NECT provided no evidence of a ureteric stone or alternative diagnosis and if the patient still complained of flank pain, contrast-enhanced CT (CECT) was performed for evaluation of an alternative diagnosis other than nephrolithiasis. If the urinalysis did not reveal microscopic hematuria or a urine specimen was not obtained,other laboratory blood test results were reported. If the patient could not void when asked by the treating physician "Could you void right now?", NECT or CECT was performed according to the discretion of the treating physician based on the clinical presentation.

\section{Ultrasonography group}

If the treating physician thought that the patient's clinical presentation was compatible with nephrolithiasis, we immediately administered parenteral analgesics and performed BUS to detect the presence of hydronephrosis. If hydronephrosis was detected, we performed NECT without waiting for the urinalysis results. The remaining protocol was the same as that for non-ultrasonography group (NUSG).

\section{Pain control protocol}

Pain was managed with parenteral analgesics for both groups according to our institution's protocol..$^{11}$ If the patient complained of pain greater than numeric rating scale (NRS) 4, ketorolac 30 $\mathrm{mg}$ and cimetropium $5 \mathrm{mg}$ were injected intravenously. Thirty minutes later, pain was reassessed by the treating physician or nurse, and if the patient still complained of pain greater than NRS 4, tramadol 50 mg was injected intravenously. After another 30 minutes, pain was reassessed by the treating physician or nurse, and if the patient still complained of intractable pain greater than NRS 4, meperidine $25 \mathrm{mg}$ was injected intravenously. Finally, after another 30 minutes, pain was reassessed by the treating physician or nurse, and if the patient still complained of intractable pain greater than NRS 4, meperidine $50 \mathrm{mg}$ was injected intravenously. Intravenous hydration was performed with normal saline at an infusion rate of $125 \mathrm{~mL} / \mathrm{hr}$ to achieve normo- 
volemia in patients without congestive heart failure or chronic renal failure on dialysis. ${ }^{11}$

Patients were enrolled between October 2014 and December 2014. During this period, if a patient experienced flank pain that the treating physician thought was compatible with the clinical features of nephrolithiasis, ED residents or the attending physicians obtained informed consent and randomized the patients to one of the two diagnostic protocols (NUSG or ultrasonography group [USG]). Randomization was performed using the 'True Random Number Generator' (http://www.random.org). Due to the nature of this study, both the treating physician and patient could not be blinded; therefore, they were aware of the allocation protocol.

Patients were excluded for the following reasons: $(1)<15$ years old, (2) pregnancy or positive urine human chorionic gonadotropin test in the ED, (3) body temperature $>37.2^{\circ} \mathrm{C}$, (4) trauma-related pain, (5) recent nephrolithiasis diagnosis, (6) unstable vital signs (e.g., initial systolic blood pressure $<90 \mathrm{mmHg}$ ) requiring immediate resuscitation, and (7) refusal of informed consent.

All ultrasonography examinations were performed at the bedside with a Sonosite I80Plus (Sonosite, Bothell, WA, USA). A 2 to $5 \mathrm{MHz}$ curved probe was used, and only the presence of hydronephrosis was recorded. Ultrasounds were performed and interpreted by six ED attending physicians and two ED residents with 2 to 14 years of point-of-care ultrasound experience. Prior to study initiation, they underwent a 2-hour bedside renal ultrasound training session conducted by a lecturer certified by the Emergency and Critical Care Imaging Society.

We did not try to determine the presence and location of the ureteric stone. Hydronephrosis was graded on a 4-point scale, ranging from mild to severe; the presence of hydronephrosis was determined by comparing the grade with that of the contralateral kidney. A normal grade was considered to indicate the absence of
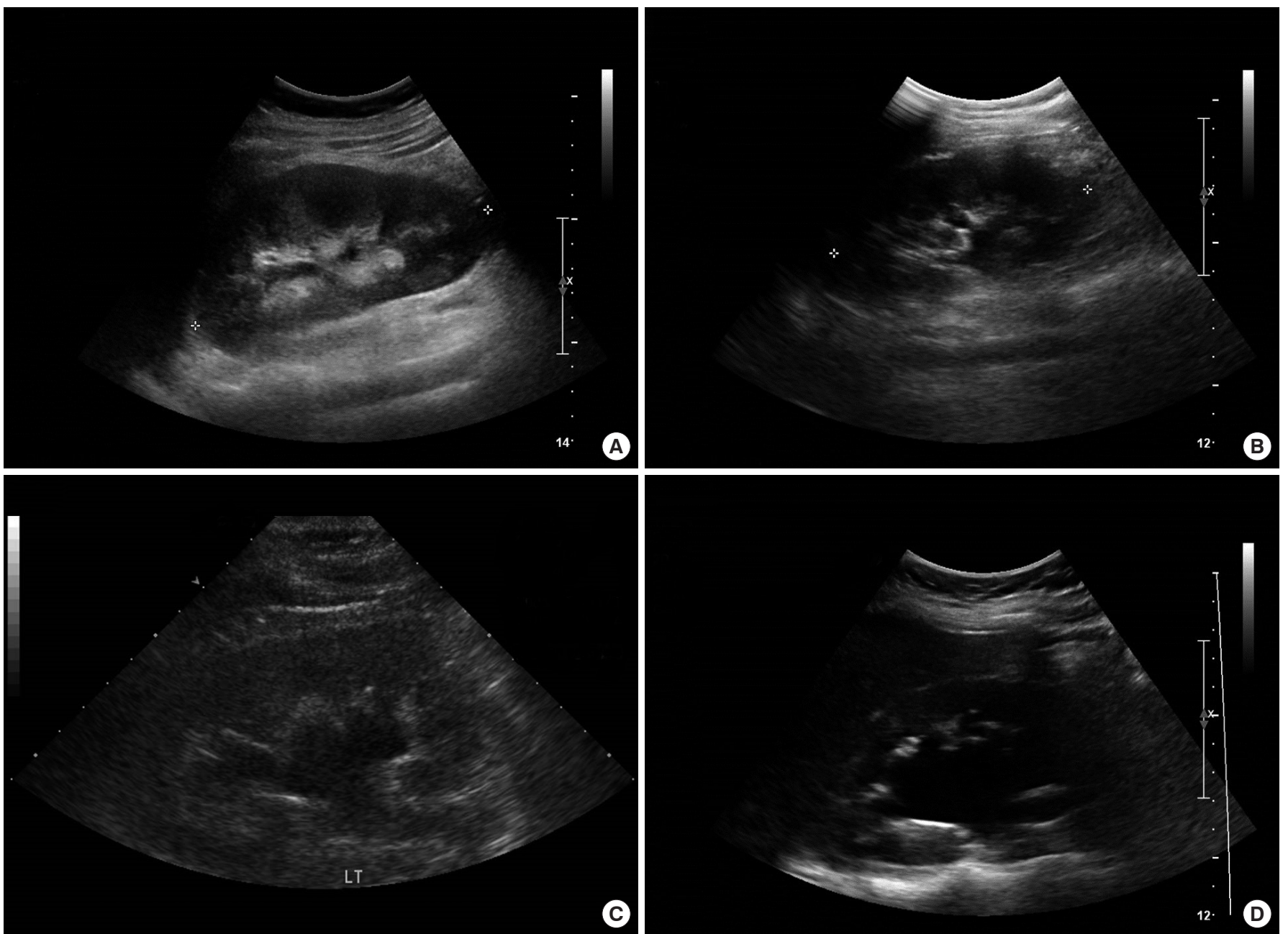

Fig. 1. Grading of hydronephrosis. (A) Grade 0, normal: no dilatation, calyceal walls are apposed to each other. (B) Grade 1, mild: dilatation of the renal pelvis without dilatation of the calyces. (C) Grade 2, moderate: moderate dilatation of the renal pelvis and calyces. (D) Grade 3, severe: gross dilatation of the renal pelvis and calyces. 
hydronephrosis (Fig. 1). If the physician could not differentiate between normal or mild grade, the results were recorded as a normal grade and absence of hydronephrosis. ${ }^{12}$

CT was performed using a 16-MDCT (Brilliance 16; Philips Healthcare, Eindhoven, The Netherlands). The tube current was automatically modulated, and the tube voltage was $120 \mathrm{kVp}$. The other parameters were as follows: anatomical range, diaphragm to the symphysis pubis; detector collimation, $1.5 \mathrm{~mm}$; rotation speed, 0.5 seconds; and pitch, 1.4 or 0.9 . Reconstructions consisted of 2-mm transverse and coronal sections. For CECT, three IV contrast agents were used: iohexol (Omnipaque 350; GE Healthcare, Princeton, NJ, USA), iopamidol (Pamiray 370; Dongkook Pharmaceutical, Seoul, Korea) and iodixanol (Visipaque 270, GE Healthcare). The IV contrast agent was administered using a mechanical injector (Medrad, Pittsburgh, PA, USA) at a rate of 3.0 $\mathrm{mL} / \mathrm{sec}$ and a dose of $2 \mathrm{~mL} / \mathrm{kg}$ body weight, and CT data acquisition was initiated 60 seconds after the start of the injection. No oral or rectal contrast material was administered to any patient.

CT was primarily interpreted by the treating physician or the on-duty faculty. For uncertain diagnoses, an immediate telephone consultation was conducted with an experienced abdominal radiologist during normal working hours or with the radiology resident during off-duty hours.

Assessment of patient disposition was at the discretion of the treating physicians.

\section{Outcomes}

The primary outcome was ED length of stay. The secondary outcomes were radiation exposure, amount of analgesics, proportion of patients with diseases other than ureteral calculus, and proportion of patients with unexpected ED revisits within 7 days from the index visit.

ED length of stay was calculated based on the difference between the initial time of the ED visit and ED departure using electronic medical records. The radiation exposure dose was defined as the sum of effective doses for all imaging studies that were performed during the ED stay. The radiation dose for CT examination was calculated based on the dose-length product reported for each CT scan, which we converted to an effective dose using the following formula: $\mathrm{mSv}=$ dose length product $(\mathrm{mGy} \cdot \mathrm{cm})$ $x$ conversion factor $0.015\left(\mathrm{mSv} \cdot \mathrm{mGy}^{-1} \cdot \mathrm{cm}^{-1}\right){ }^{13}$ For plain radiographs (chest, abdomen, kidney, ureter, and bladder), we calculated effective doses using previously estimated doses for each type of examination. ${ }^{14}$ The amount of analgesics was defined as the grade of analgesics according to the different grades of the analgesic protocol. The proportion of patients with diseases other than ureteral calculus was ascertained according to the final diagnosis of the treating physician. The proportion of patients with unexpected ED revisits within 7 days from the index visit was determined primarily by reviewing our hospital's electronic medical records. For patients lost to outpatient follow up, we conducted a telephone follow-up survey. If patients visited the ED at other hospitals within 7 days from the index visit, these cases were also included as unexpected ED revisits.

\section{Statistical methods}

Normally distributed continuous variables are expressed as means and standard deviations. Continuous variables that were not normally distributed are expressed as medians and interquartile ranges. Independent t-tests were used to compare age between the two groups. $\chi^{2}$ tests were used to compare sex, pain location, presence of costovertebral angle tenderness, characteristics of pain, and amount of analgesics. Fisher's exact tests were used to assess additional CECT, proportion of patients with diseases other than ureteral calculus, and proportion of patients with unexpected ED revisits. Other variables were compared using Mann Whitney U-tests.

Because we could not obtain previous data to estimate the sample size, this study was conducted as a 3-month pilot trial. We considered a P-value $<0.05$ to be statistically significant. Statistical analysis was performed using PASW Statistics ver. 18.0 (SPSS Inc., Chicago, IL, USA).

\section{RESULTS}

Between October 2014 and December 2014, a total of 176 patients visited the ED with a complaint of flank pain. Of these patients, 149 patients were enrolled in the study and underwent randomization. After excluding the patients who did not provide informed consent, 103 patients were included in the analysis (NUSG, 51; USG, 52) (Fig. 2). There were no significant differences between the two groups in terms of sex, age, vital signs, and variables of pain (Table 1).

ED length of stay was shorter for the USG than for the NUSG (89 and 163 minutes, respectively; $P<0.001$ ). The total amount of radiation during the ED visit (NUSG, 5.29 mSv; USG, $5.08 \mathrm{mSv}$, respectively; $P=0.392$ ) and the proportion of patients who underwent contrast-enhanced CT (NUSG, 13.0\%; USG, 4.5\%, respectively; $\mathrm{P}=0.267$ ) were not significantly different. The amount of analgesics according to the different stages of pain control protocol was not significantly different between the two groups $(P=0.341)$. The proportion of patients with diseases other than ureteral calculus was also not different between the two groups (NUSG, 13.0\%; USG, 6.8\%, respectively; $P=0.486$ ). The proportion of patients with unexpected ED revisits was not significantly 


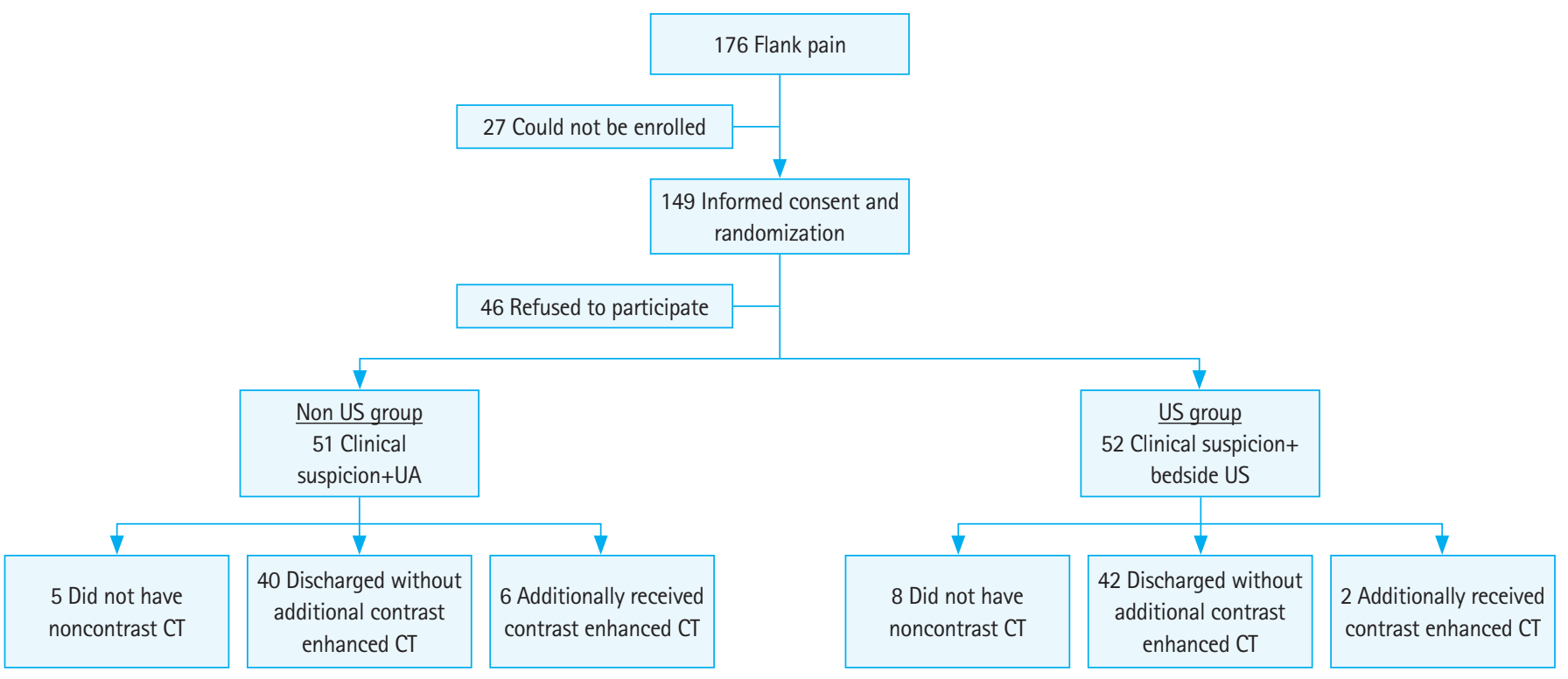

Fig. 2. Patients selection flow diagram. US, ultrasonography; UA, urinalysis; CT, computed tomography.

Table 1. General demographics of the study participants

\begin{tabular}{|c|c|c|c|}
\hline & NUSG $(n=51)$ & USG $(n=52)$ & P-value \\
\hline No. of men & $33(64.7)$ & $35(67.3)$ & 0.780 \\
\hline Age (yr) & $44.55 \pm 14.56$ & $46.63 \pm 10.58$ & 0.407 \\
\hline Systolic arterial pressure (mmHg) & $130.0(130.0-160.0)$ & $130.0(130.0-140.0)$ & 0.941 \\
\hline Diastolic arterial pressure $(\mathrm{mmHg})$ & $80.0(70.0-90.0)$ & $80.0(80.0-90.0)$ & 0.833 \\
\hline Respiratory rate & $20.0(20.0-20.0)$ & $20.0(20.0-20.0)$ & 0.570 \\
\hline Body temperature $\left({ }^{\circ} \mathrm{C}\right)$ & $36.00(36.00-36.50)$ & $36.50(36.00-36.50)$ & 0.413 \\
\hline Pain location & & & 0.367 \\
\hline Right flank & $13(25.5)$ & $8(15.4)$ & \\
\hline Right abdomen & $1(2.0)$ & $1(1.9)$ & \\
\hline Right flank and abdomen & 19 (37.3) & $18(34.6)$ & \\
\hline Left flank & $9(17.6)$ & $16(30.8)$ & \\
\hline Left abdomen & $0(0.0)$ & $2(3.8)$ & \\
\hline Left flank and abdomen & $9(17.6)$ & $7(13.5)$ & \\
\hline Presence of CVAT & $44(86.3)$ & $44(84.6)$ & 0.811 \\
\hline Characteristics of pain & & & 0.369 \\
\hline Colicky & $31(60.8)$ & $36(69.2)$ & \\
\hline Continuous & $20(39.2)$ & $16(30.8)$ & \\
\hline Presence of nausea & $19(37.3)$ & $24(46.2)$ & 0.360 \\
\hline Presence of vomiting & $7(13.7)$ & $13(25.0)$ & 0.148 \\
\hline Presence of hematuria & $43(84.3)$ & 47 (90.3) & 0.840 \\
\hline
\end{tabular}

Values are presented as number (\%), mean \pm standard deviation, or median (interquartile range).

NUSG, non-ultrasonography group; USG, ultrasonography group; CVAT, costovertebral angle tenderness.

different between the two groups (NUSG, 7.8\%; USG, 9.6\%, respectively; $P=1.000$ ) (Table 2).

\section{DISCUSSION}

This study showed that the use of BUS reduced ED length of stay by about 74 minutes, without increasing unexpected ED revisits.
To the best of our knowledge, this study is the first to demonstrate the role of BUS in terms of reducing the ED length of stay.

Although there is an ongoing debate regarding the first choice of primary imaging, NECT is recognized as the primary imaging modality due to its high sensitivity and specificity. Recently, SmithBindman et al. ${ }^{6}$ documented that BUS, rather than CT, as an initial imaging modality was associated with significantly less radiation, 
Table 2. Comparison of variables between the two groups

\begin{tabular}{|c|c|c|c|}
\hline & NUSG $(n=51)$ & USG $(n=52)$ & P-value \\
\hline Symptom duration (min) & $180.0(60.0-270.0)$ & $120.0(60.0-247.5)$ & 0.325 \\
\hline Proportion of patients who underwent additional contrast-enhanced $\mathrm{CT}^{\mathrm{a}}$ ) & $6 / 46(13.0)$ & $2 / 44(4.5)$ & 0.267 \\
\hline Grade of analgesic protocol & & & 0.341 \\
\hline 0 & $0(0)$ & $1(1.9)$ & \\
\hline 2 & 5 (9.8) & $10(19.2)$ & \\
\hline 3 & $17(33.3)$ & $21(40.4)$ & \\
\hline 4 & $1(2.0)$ & $5(9.6)$ & \\
\hline Proportion of patients with diseases other than ureteral calculus ${ }^{\mathrm{a})}$ & $6 / 46(13.0)$ & $3 / 44(6.8)$ & 0.486 \\
\hline \multicolumn{4}{|l|}{ Grade of hydronephrosis on bedside ultrasonography } \\
\hline Urinalysis turn-around time & $78.0(58.0-108.0)$ & $76.0(51.0-133.5)$ & 0.790 \\
\hline Proportion of patients with unexpected ED revisits & $4(7.8)$ & $5(9.6)$ & 1.000 \\
\hline
\end{tabular}

Values are presented as median (interquartile range) or number (\%).

NUSG, non-ultrasonography group; USG, ultrasonography group; ED, emergency department; CT, computed tomography; NA, not applicable.

a) Excluding patients who did not undergo CT (NUSG, 46; USG, 44).

with little difference in patient outcome. However, approximately $40 \%$ of patients in the US group underwent an additional CT scan. These findings reflect conditions in the field where NECT needs to be performed, despite exposure to radiation as the biggest drawback of NECT. This may stem from reduced physician confidence in BUS findings and difficulties in evaluating ureteral calculi. This implies that a lack of hydronephrosis on BUS cannot serve as the basis to determine patient disposition.

In the present study, the patients presumed to have nephrolithiasis and with positive ultrasound findings were selected to undergo NECT at the bedside, and this resulted in meaningful reductions in ED length of stay. However, unexpectedly, CT was conducted approximately 2.5 times more frequently for the NUSG than the USG, although this was not statistically significant. The average radiation dose was approximately $5.08 \mathrm{mSv}$ for the USG, compared with approximately $5.29 \mathrm{mSv}$ for the NUSG. If urinalysis revealed no microscopic hematuria, NECT should have been performed first; then, according to the result, CECT should be performed for alternative diagnoses. In practice, however, CECT is performed first, based on the judgment of the treating physician with respect to the clinical presentation in some cases, which is related with low confidence. Therefore, even if NECT was used as the diagnostic imaging modality for nephrolithiasis, conducting BUS prior to NECT reduced radiation exposure.

Our study had several limitations. First, the study duration was short; only the results from a 3-month flank pain cohort were examined. Hence, the effectiveness of the methods needs to be verified based on data from long-term comparative studies that estimate the most appropriate sample size. Second, owing to the unblinded nature of the study, healthcare professionals may have subconsciously discharged the patients in the USG prematurely. Despite no difference between the two groups in terms of unexpected ED revisits, researcher bias may have been present, because the degree of pain felt by individual patients over time was not measured. Third, measurement bias might have been present, because hydronephrosis on BUS was exclusively determined by those who performed the ultrasonography. Therefore, the decision regarding the presence of hydronephrosis on BUS might have been affected by the sonographer. Also, additional bias might have been present because the researchers obtained the clinical information and conducted the physical examinations during the study. Fourth, although the ED length of stay was somewhat reduced, factors affecting the reduction in overall ED overcrowding and level of patient satisfaction were not assessed. Fifth, we only observed the presence of hydronephrosis; thus, these findings could not be extrapolated to other scenarios that are associated with acute flank pain. Last, because cost-effectiveness analysis was not a concern in this study, we could not determine the cost-effectiveness of BUS for patients with renal colic.

In conclusion, the use of BUS reduced the ED length of stay by about 74 minutes, without increasing unexpected ED revisits. 


\section{CONFLICT OF INTEREST}

No potential conflict of interest relevant to this article was reported.

\section{REFERENCES}

1. Fwu CW, Eggers PW, Kimmel PL, Kusek JW, Kirkali Z. Emergency department visits, use of imaging, and drugs for urolithiasis have increased in the United States. Kidney Int 2013; 83:479-86.

2. Noble VE, Brown DF. Renal ultrasound. Emerg Med Clin North Am 2004;22:641-59.

3. Scales CD Jr, Smith AC, Hanley JM, Saigal CS; Urologic Diseases in America Project. Prevalence of kidney stones in the United States. Eur Urol 2012;62:160-5.

4. Hyams ES, Korley FK, Pham JC, Matlaga BR. Trends in imaging use during the emergency department evaluation of flank pain. J Urol 2011;186:2270-4.

5. Sinclair D, Wilson S, Toi A, Greenspan L. The evaluation of suspected renal colic: ultrasound scan versus excretory urography. Ann Emerg Med 1989;18:556-9.

6. Smith-Bindman R, Aubin C, Bailitz J, et al. Ultrasonography versus computed tomography for suspected nephrolithiasis. N Engl J Med 2014;371:1100-10.

7. Niemann T, Kollmann T, Bongartz G. Diagnostic performance of low-dose CT for the detection of urolithiasis: a meta-analysis. AJR Am J Roentgenol 2008;191:396-401.

8. Portis AJ, Sundaram CP. Diagnosis and initial management of kidney stones. Am Fam Physician 2001;63:1329-38.

9. Varanelli MJ, Coll DM, Levine JA, Rosenfield AT, Smith RC. Relationship between duration of pain and secondary signs of obstruction of the urinary tract on unenhanced helical CT. AJR Am J Roentgenol 2001;177:325-30.

10. Elton TJ, Roth CS, Berquist TH, Silverstein MD. A clinical prediction rule for the diagnosis of ureteral calculi in emergency departments. J Gen Intern Med 1993;8:57-62.

11. Teichman JM. Clinical practice. Acute renal colic from ureteral calculus. N Engl J Med 2004;350:684-93.

12. Dalziel PJ, Noble VE. Bedside ultrasound and the assessment of renal colic: a review. Emerg Med J 2013;30:3-8.

13. Huda W, Ogden KM, Khorasani MR. Converting dose-length product to effective dose at CT. Radiology 2008;248:995-1003.

14. Mettler FA Jr, Huda W, Yoshizumi T, Mahesh M. Effective doses in radiology and diagnostic nuclear medicine: a catalog. Radiology 2008;248:254-63. 